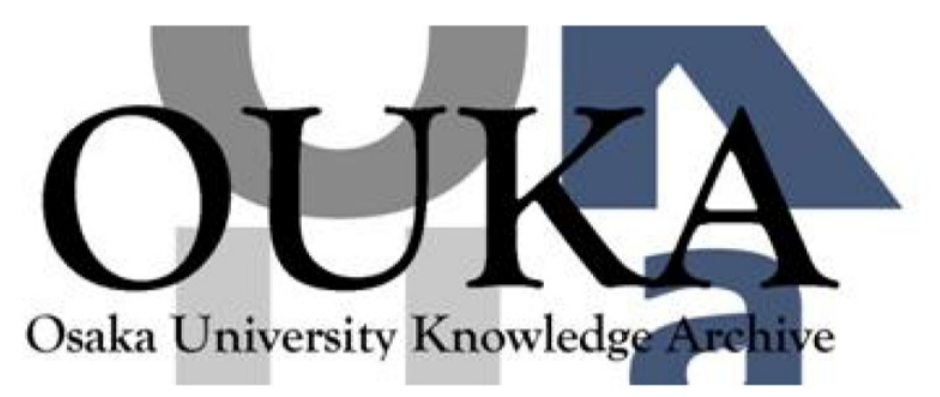

\begin{tabular}{|c|l|}
\hline Title & $\begin{array}{l}\text { Chemically Reactive Species In Liquids } \\
\text { Generated By Atmospheric-Pressure Plasmas And } \\
\text { Their Roles In Plasma Medicine }\end{array}$ \\
\hline Author(s) & Hamaguchi, Satoshi \\
\hline Citation & AIP Conference Proceedings. 1545 p. 214-p. 222 \\
\hline Issue Date & $2013-07-11$ \\
\hline oaire:version & AM \\
\hline URL & https://hdl. handle. net/11094/78706 \\
\hline rights & \\
\hline Note & \\
\hline
\end{tabular}

Osaka University Knowledge Archive : OUKA

https://ir. Library. osaka-u. ac. jp/

Osaka University 


\title{
Chemically Reactive Species In Liquids Generated By Atmospheric-Pressure Plasmas And Their Roles In Plasma Medicine
}

\author{
Satoshi Hamaguchi \\ Center for Atomic and Molecular Technologies, Graduate School of Engineering, Osaka University, \\ 2-1 Yamadaoka, Suita, Osaka 565-0871, Japan
}

\begin{abstract}
Plasmas whose gas temperatures are close to room temperature may be generated in ambient air or a gas at atmospheric pressure with the use of low-frequency high voltage or lowpower radio-frequency (RF) or microwave power applied to electrodes. Such plasmas can serve as a powerful source of free radicals and/or chemically reactive species that arise from atoms and molecules of the ambient gas. Recently use of such plasmas for medical purposes has attracted much attention as they can be implemented in possible medical devices that can cause blood coagulation, heal wounds, facilitate angiogenesis, sterilize surgical devices as well as living tissues without harming healthy cells, and selectively inactivate cancer cells. Especially of interest among reactive species generated by atmospheric-pressure plasmas (APP) are reactive oxygen species (ROS) and reactive nitrogen species (RNS) that are generated in liquid phase. Since most living tissues and cells are immersed in liquids (such as blood or culture media), reactive species generated by APPs in the gas phase are transported to the liquid phase and possibly converted to different types of reactive species therein before causing some influence on the tissues or cells. In this study, the rate equations are solved to evaluate concentrations of various reactive species in pure water that are originated by plasma reactions in atmosphere and possible effects of such species (including ROS/RNS) on living tissues and cells are discussed.
\end{abstract}

\section{INTRODUCTION}

Low temperature plasmas have been applied to a wide range of industrial processes such as fabrication of micro and nano-scale structures on the surfaces of semiconductor chips and thin film deposition for surface coating of various materials. Recently, as one of such applications, use of low-temperature plasmas for medical treatment and material processing for medical applications has attracted much attention in the plasma research community. In this article, the recent trend and progresses in this field, known collectively as "plasma medicine," is reviewed.

In general, research in plasma medicine may be categorized into three subfields. One of them is surface modification/treatment of biomedical materials [1-3]. For example, surfaces of petri dishes (made of polystyrene) are typically pretreated by oxygen plasmas. Surface coating of metal artificial joints by hydroxyapatite is often done with plasma spraying with the use of thermal plasmas. Surface polymerization 
by plasma CVD (chemical vapor deposition) is also widely studied for the formation of biocompatible films.

Another subfield that is also widely studied is plasma sterilization [4-6]. Including commercial products such as plasma-based air purifiers, this subfield has seen much technological development in recent years in areas beyond medical equipment although the fundamental mechanisms of bactericidal effects by plasmas have yet to be understood better. Furthermore, there are other sources for infectious diseases that are still difficult to remove by plasmas, such as spore forming bacteria, viruses, and prions, and inactivation of them is still a topic of active research in this subfield. Usually difficulty of treating such infection sources by plasmas lies in the fact that, in reality, removal of them needs to be done without damaging the environments (such as human bodies or medical equipment) where they exist, and often in a cost effective manner.

The third but not least subfield of the three is study on therapeutic or clinical use of plasmas. This subfield is often considered as plasma medicine in narrow sense. Probably the best known plasma device that is already in use for surgical operations is argon plasma coagulator (APC) [7-9]. For this device, heat generated by Ar plasma is used for cauterization or thermal ablation of tissues. In this sense, the APC is similar to an electrical scalpel. The essential difference between them is that the tip of an APC device is contactless with tissues.

In current research of the above subfield, however, low-temperature, noncauterizing plasma devices have been much of the focus for scientific and technological development. Recent studies have shown that application of lowtemperature plasmas directly to living cells and tissues has some therapeutic effects such as blood coagulation, angiogenesis, prevention of organ adhesion, and wound healing [10]. The origin of such effects is considered to be reactive species generated by plasmas. Under typical discharge conditions in air, plasma efficiently generates reactive oxygen species (ROS) /reactive nitrogen species (RNS) such as atomic oxygen $\mathrm{O}$, hydroxyl radical $\mathrm{OH}$, hydroperoxyl (or perhydroxyl) radical $\mathrm{HOO}$, singlet oxygen ${ }^{1} \mathrm{O}_{2}$, and nitric oxide (or nitrogen monoxide) NO. Such chemically reactive species are often generated from endogenous sources in living bodies and play crucial roles in controlling cell signaling pathways and maintaining homeostasis. In this sense, perhaps it is not surprising that externally generated ROS/RNS by plasmas can have some significant effects on living cells and tissues under certain conditions. In what follows, some recent results that the author and his collaborators have obtained in their study on medical application of plasmas are presented [11].

\section{CELL PROLIFERATION BY PLASMA APPLICATION}

It has been known that chemically reactive species generated by atmosphericpressure plasmas (APPs) can enhance cell proliferation. As an example of orthopedic application of plasma effects, effects of APP application on growth of mesenchymal stem cells and other cells that are of interest in orthopedics have been examined. In the experiments, three conditions were tested. In the first condition, low-temperature APPs were directly injected into a culture medium [Dulbecco's Modified Eagle Medium (DMEM) with fetal bovine serum (FBS)] containing cells and the cells were 
cultured in the same medium for a few days. In the second condition, immediately after the medium containing cells was exposed to a plasma, the plasma-exposed medium was discarded and replaced with a fresh medium of the same kind and the cells were cultured for a few days in the new medium. In the third conditions, DMEM without cells was exposed to a plasma first and then the cells were cultured in the plasma treated medium for a few days. In each case, cell proliferation (or cell death in the case of overexposure to plasma) was observed, which indicates that the presence of either chemically reactive species dissolved in the medium or solutes modified by such chemically reactive species affects cell viability.

The plasma device that we used for these experiments is schematically depicted in Fig. 1. It is a low-frequency plasma jet device driven typically by $27 \mathrm{kHz}$ and (peak-topeak) $10 \mathrm{kV}$ sinusoidal voltage with a $\mathrm{He}$ gas flow of $3 \mathrm{~L} / \mathrm{min}$ at room temperature [12]. The tip of plasma (i.e., an extended discharge emitting visible light) is set to touch the surface of $100 \mu \mathrm{L}$ DMEM that is contained in a well of a 96 well plate.

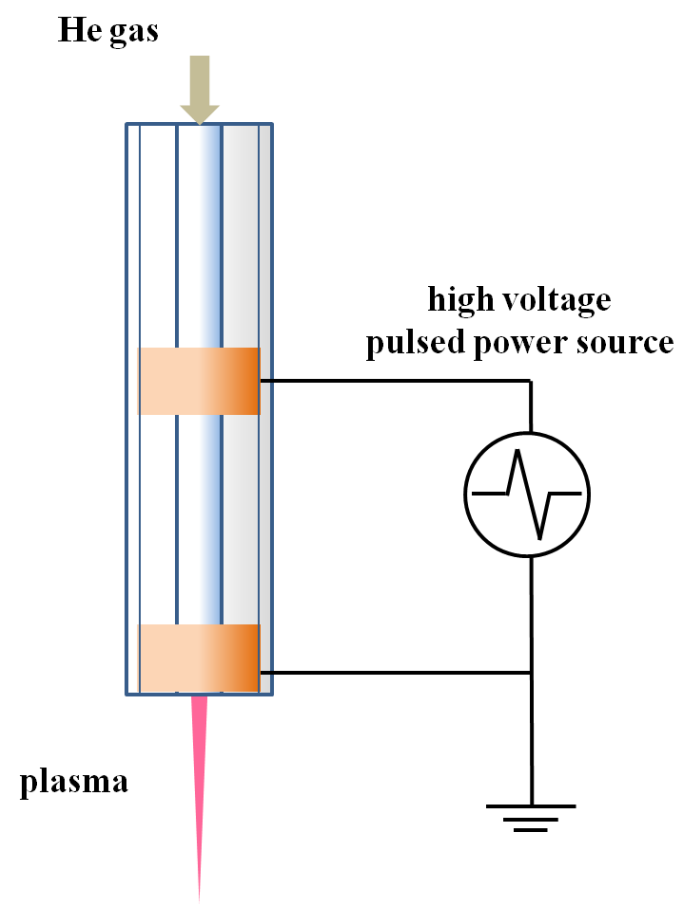

FIGURE 1. Schematic diagram of a low-frequency plasma jet system. For cell viability and proliferation assays that we performed, the device was driven typically by $27 \mathrm{kHz}$ and (peak-to-peak) $10 \mathrm{kV}$ sinusoidal voltage with a $\mathrm{He}$ gas flow of $3 \mathrm{~L} / \mathrm{min}$ at room temperature [12].

Figure 2 shows an example of the results of a cell viability and proliferation assay [12]. In this assay, $100 \mu \mathrm{L}$ DMEM was placed in a well of a 96 well plate and 6000 cells of Human Synoviocytes (HS) were seeded per well. In each 96 well plate, only a couple of dozens of wells were used for cell seeding and those wells were sufficiently separated from each other, so that plasma jet injection into a single well would not 
affect cells in neighboring wells. After each well was exposed to plasma jet for a certain period of time (which varied from 0 to $90 \mathrm{sec}$.), cells were cultivated for 6 hours in $\mathrm{CO}_{2}$ incubator and then the number of cells were counted by a WST (Water soluble Tetrazolium salts)-8 assay. It is seen that exposure to an APP for a limited period can enhance cell proliferation. The level of free radical generation in the medium was examined by dROMs tests [13] and the correlation between cell proliferation and oxidative stress was observed.

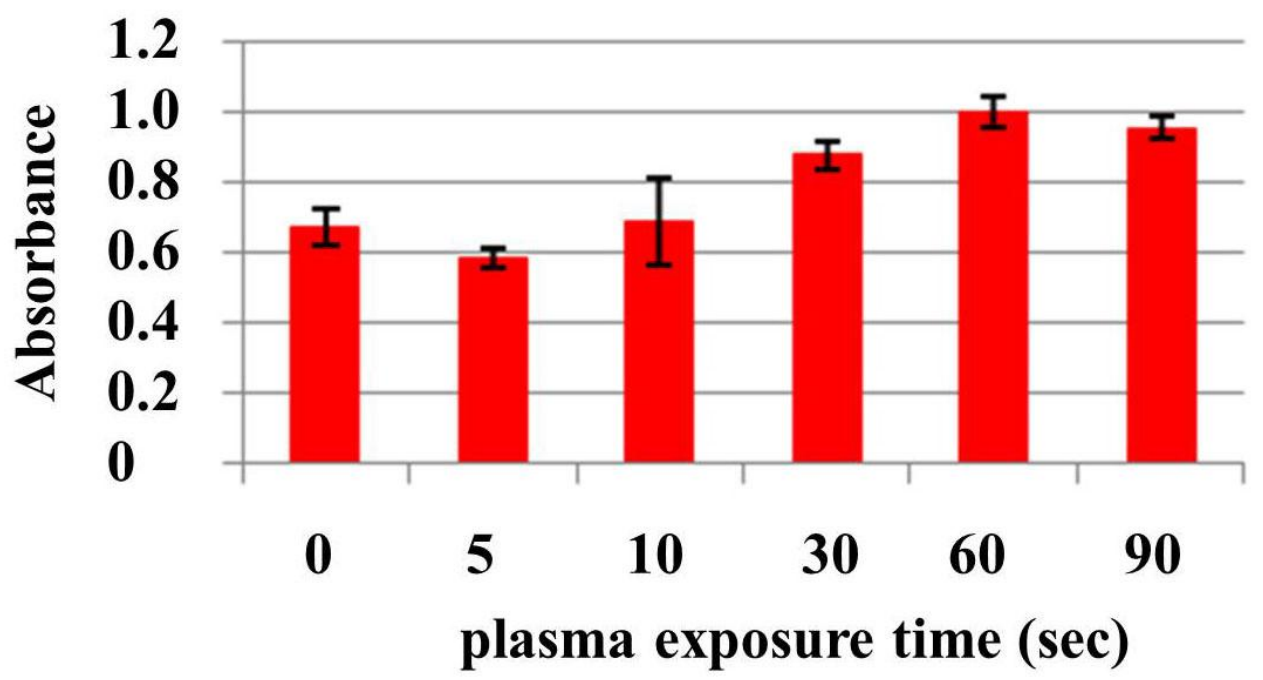

FIGURE 2. Results of a cell viability and proliferation assay for Human Synoviocytes (HS)[12]. It is seen that exposure to an APP for a limited period can enhance cell proliferation.

\section{REACTIVE SPECIES IN LIQUIDS}

\section{Background}

Cell proliferation effects seen in Fig. 2 are also considered to be caused by reactive species in liquid (DMEM) generated by plasmas. For therapeutic use of plasmas such as wound healing and prevention of organ adhesion, as mentioned earlier, when a living tissue is exposed to a low-temperature APP, there is almost always a liquid layer, such as blood, lymph, and interstitial fluid, that separates the gas phase from the tissue. Therefore, in both cell proliferation assays and therapeutic use of APPs, chemically reactive species generated by a plasma discharge in the gas phase need to be transported through the liquid phase before reacting with the tissue surfaces. During this transport process, some of the chemically reactive species may react with other species and, therefore, the observation of gas-phase chemically reactive species does 
not directly convey information on abundant chemically reactive species in the vicinity of the tissue.

In this section, we discuss the growth and decay processes of chemically reactive species in liquid facing an APP. For the sake of simplicity, we only consider pure water as a liquid exposed to an APP in this study.

\section{Simulation Model}

We have developed a simplified computational model that governs interactions of various chemical species in water facing a gas plasma. The conceptual diagram of the system we consider in this study is shown in Fig. 3. It is assumed that a plasma is generated in air whose composition is constant in time (due to efficient ventilation). Charged species as well as other chemically reactive charge-neutral species that are generated by plasma discharge are assumed to be uniformly distributed in the gas phase. The gas phase species are assumed to be in steady state and, at time $t=0$, they are assumed to be exposed to water suddenly. Charge neutral species are assumed to enter water at their thermal velocities whereas charged species are assumed to enter water following the (ambipolar) field so that the charge neutrality of water should be maintained. In this study, we use data of gas phase species from published literatures (such as [14]).

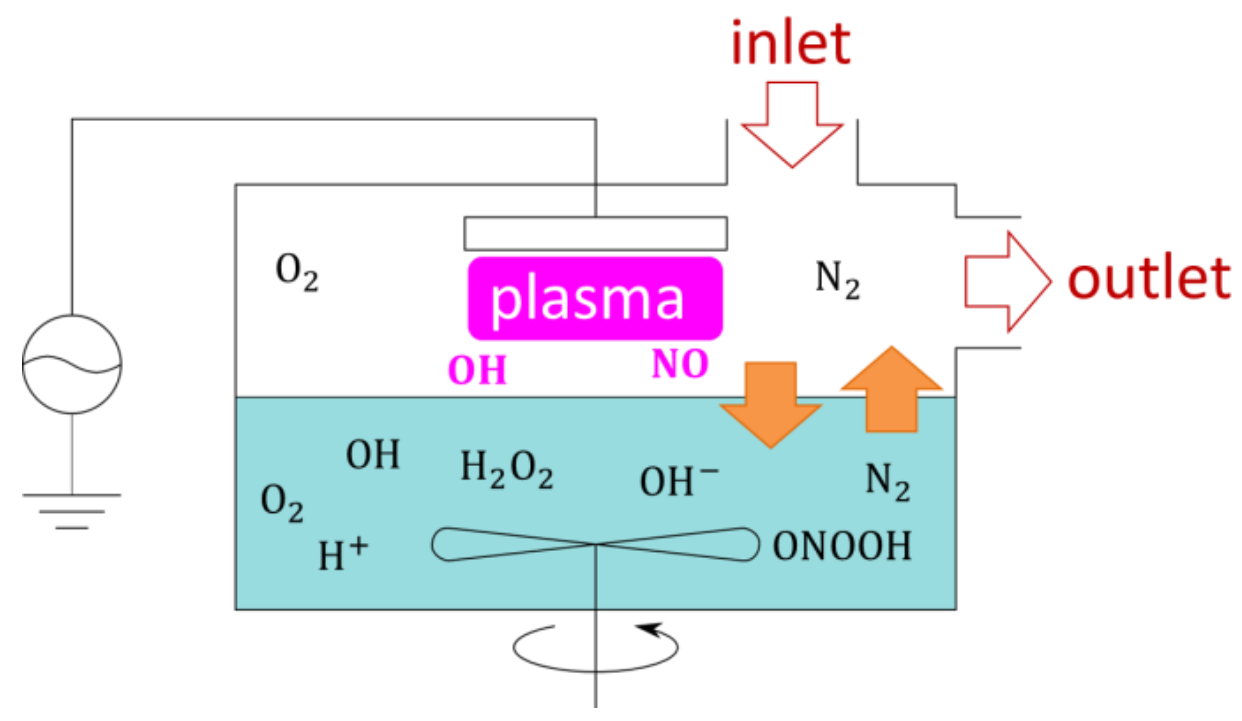

FIGURE 3. Conceptual diagram of the system

Under the assumption that water is well mixed (as indicated in Fig. 1), diffusion of chemical species in water is not taken into account in this study. We have used a zerodimensional simulation model for chemical reactions in water. The nonlinear (and typically stiff) ordinary differential equations of chemical reactions among multiple species were solved with a semi-implicit method. 


$$
Y(t+\Delta t)=Y(t)+\Delta t \cdot A^{-1} F(t)
$$

Here the function $Y(t)$ represents the vector of densities of all species at time $t$ that satisfies the nonlinear ordinary differential equation

$$
\frac{d Y}{d t}=A(Y) Y
$$

The outward flux of chemical species from water to the gas phase is determined based on the assumption that, in steady state, it balances with the influx of the same species via Henry's law. The gaseous species entering water are assumed to be dissolved into water without any barrier (e.g. with no reflection from the water surface). In other words, the sticking coefficients of gaseous species at the water surface are assumed to be unity. The rate constants of reactions are cited from NIST online database and other references on biology, pulse radiolysis, and tropospheric chemistry.

\section{Simulation Results}

Figure 4 shows the simulated densities as functions of time when only hydroxyl radicals $(\mathrm{OH})$ (as well as $\mathrm{N}_{2}$ and $\mathrm{O}_{2}$, i.e., dominant species of air) are assumed to be dissolved into water. The $\mathrm{OH}$ flux from the gas phase depends on its density there. The typical value of $\mathrm{OH}$ density in the gas phase is taken from [14] for non-thermal dielectric barrier discharge (DBD) plasma. The dissolving rate of $\mathrm{OH}$, which is defined as the number of $\mathrm{OH}$ radicals (in units of moles) supplied to the unit volume of water per unit time, is assumed to be $1.0 \times 10^{-1} \mathrm{~mol} \mathrm{~L}^{-1} \mathrm{~s}^{-1}$. It is seen in Fig. 4 that $\mathrm{OH}$ in water quickly converted to hydrogen peroxide $\mathrm{H}_{2} \mathrm{O}_{2}$ and a few other species. The dominant reaction here is the formation of $\mathrm{H}_{2} \mathrm{O}_{2}$, i.e.,

$$
2 \mathrm{OH} \rightarrow \mathrm{H}_{2} \mathrm{O}_{2}
$$

with the rate constant being $k=4.5 \times 10^{9} \mathrm{~L} / \mathrm{mol} \cdot \mathrm{s}$. The density of $\mathrm{OH}$ decays algebraically, rather than exponentially, and its half-life time (which depends on the initial density $n_{0}$ ) is given by $\mathrm{T}=1 / k n_{0}$. If the initial density is $n_{0}=10^{-6} \mathrm{~mol} / \mathrm{L}$ (as in Fig. 3), then its half-life time is about $0.2 \mathrm{~ms}$.

Figure 5 similarly shows the simulated densities as functions of time when only nitric oxide (NO) molecules (as well as $\mathrm{N}_{2}$ and $\mathrm{O}_{2}$ ) are assumed to be dissolved into water. It is seen that NO dissolves in water without being converted to other species.

Figure 6 shows the simulated densities as functions of time when both hydroxyl radicals $(\mathrm{OH})$ and nitric oxide (NO) molecules (generated by a plasma, in addition to $\mathrm{N}_{2}$ and $\mathrm{O}_{2}$ of air) are assumed to be dissolved into water. A variety of species such as nitrate ion $\left(\mathrm{NO}_{3}{ }^{-}\right)$, peroxynitrous acid $\mathrm{ONOOH}$, and superoxide radical anion $\left(\mathrm{O}_{2}{ }^{-}\right)$ are shown to be generated in water. Especially notable is that the water becomes acidic due to the generation of nitric acid, which is consistent with earlier experimental observations [2]. 


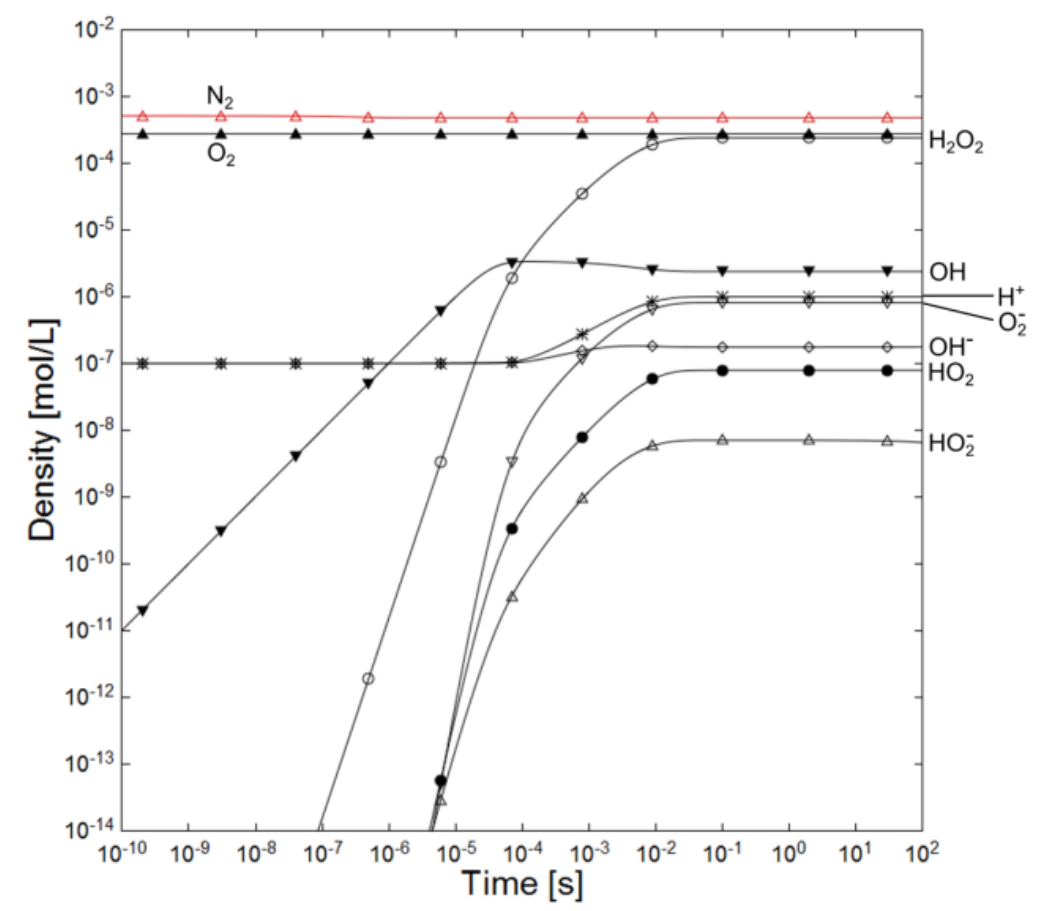

FIGURE 4. Simulated densities as functions of time; The dissolving rate of $\mathrm{OH}$ is $1.0 \times 10^{-1} \mathrm{~mol} \mathrm{~L}^{-1} \mathrm{~s}^{-1}$.

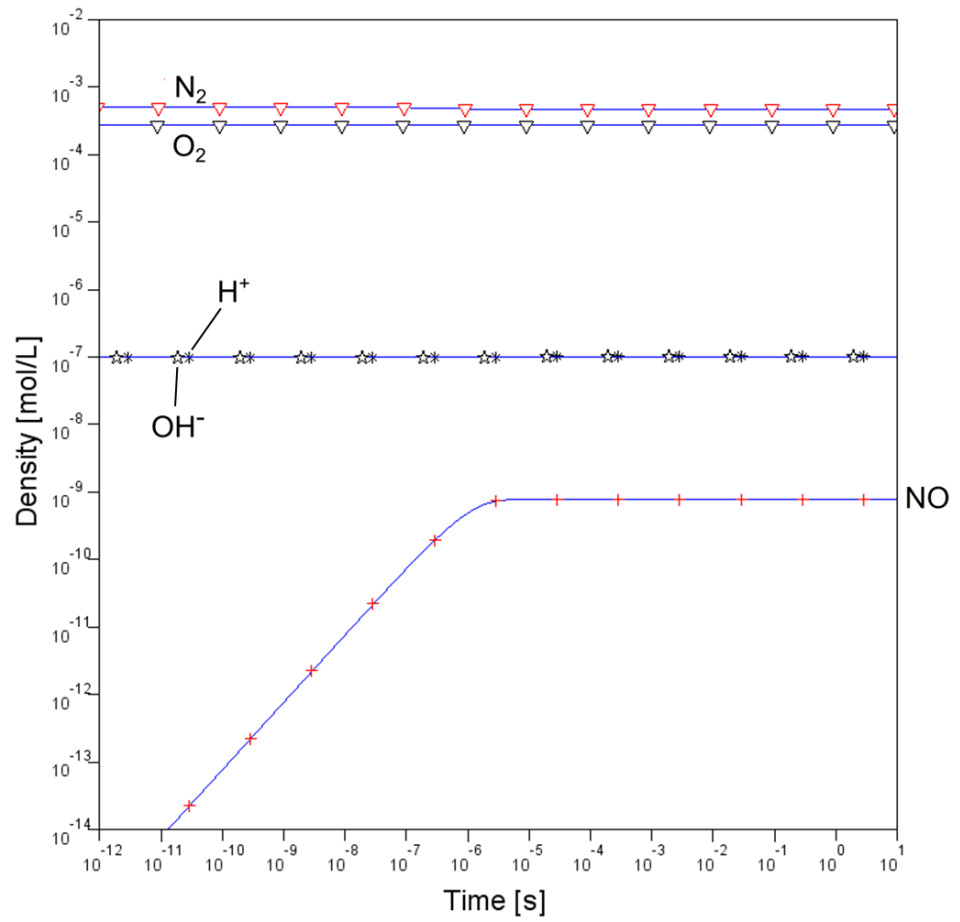

FIGURE 5. Simulated densities as functions of time; The dissolving rate of NO is $7.6 \times 10^{-4} \mathrm{~mol} \mathrm{~L}^{-1} \mathrm{~s}^{-1}$. 


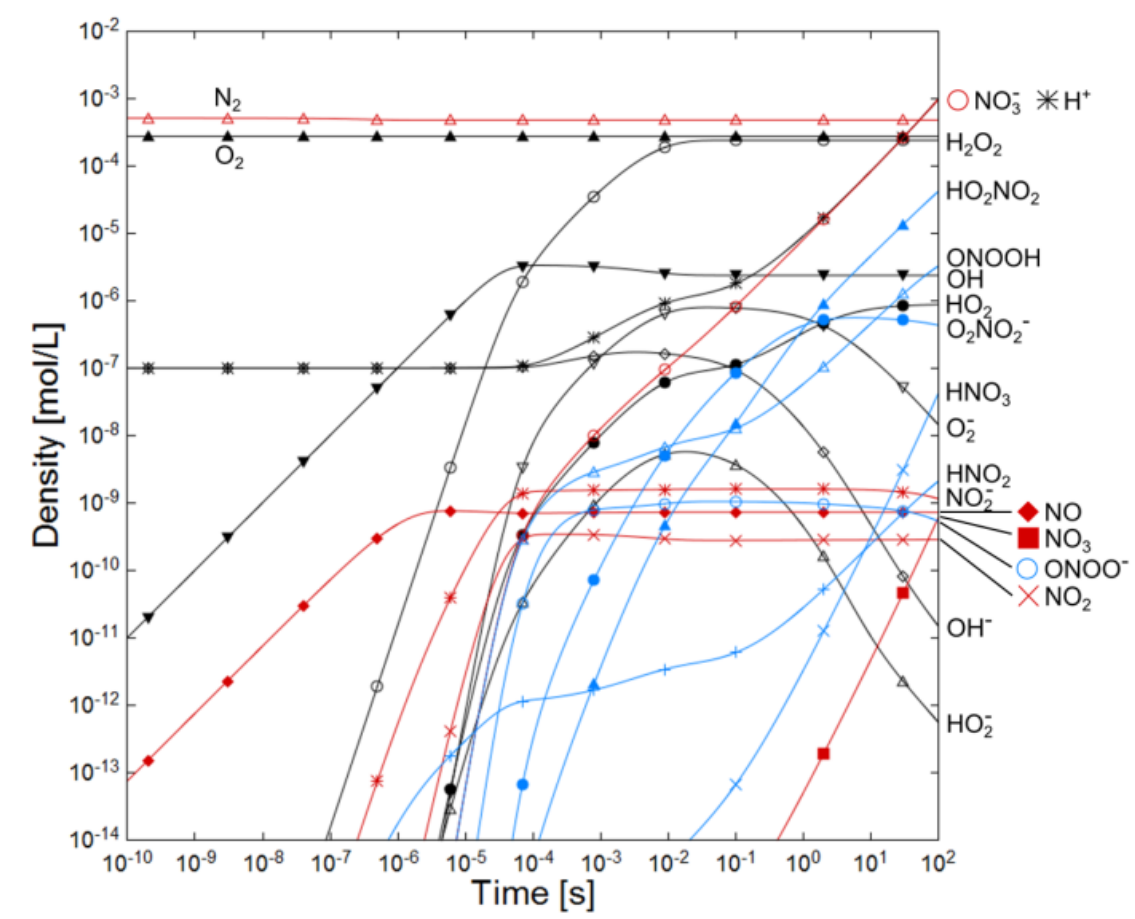

FIGURE 6. Simulated densities as functions of time. The dissolving rates are OH: $1.0 \times 10^{-1} \mathrm{~mol} \mathrm{~L}^{-1} \mathrm{~s}^{-1}$ and NO: $7.6 \times 10^{-4} \mathrm{~mol} \mathrm{~L}^{-1} \mathrm{~s}^{-1}$.

These results show that, although that dissolving of $\mathrm{OH}$ or $\mathrm{NO}$ separately results in relatively simple or even no further chemical reactions in water, combined dissolving of $\mathrm{OH}$ and $\mathrm{NO}$ generates far more chemical species, as indicated in Fig. 6.

\section{CONCLUSIONS}

When a liquid is exposed to an APP that generates various kinds of charged and charge-neutral chemically reactive species in the gas phase, many of those species dissolve into the liquid and are transformed to different types of ROS/RNS therein. It is these ROS/RNS in the liquid phase that directly affect living cells if an lowtemperature APP is applied to living tissues since there is almost always a liquid layer, such as blood, lymph, and interstitial fluid, lying between the gas phase and tissue. For cell viability proliferation assays such as those of Fig. 2, some long-lived ROS/RNS in the medium are also considered to affect cells, causing, e.g., acceleration of cell cycles. Therefore, for the understanding of how a gas plasma can affect living organisms when the liquid containing the organisms is exposed to the plasma, it is vital to know what reactive species are generated at what densities in the liquid.

In this study, we have developed a numerical code to study time evolution of various charged and charge-neutral species, including hydrogenated electrons, in pure water. Pure water was selected as the medium for the sake of simplicity. It has been 
demonstrated that, if both $\mathrm{OH}$ and NO, whose densities can be in the range of $10^{12} \sim$ $10^{14} \mathrm{~cm}^{-3}$ in the gas phase if they are generated by an APP in air, are simultaneously dissolved into water, a variety of ROS/RNS are generated in water although separate dissolving $\mathrm{OH}$ or $\mathrm{NO}$ into water results in relatively simple or even no further chemical reactions.

\section{ACKNOWLEDGMENTS}

The work has been performed in collaboration with Prof. H. Yoshikawa and Prof. A. Myoui of the School of Medicine, Osaka University. The author also acknowledges Mr. K. Masuda, Dr. Y. Moriguchi, and Dr. K. Okada, for performing the cell proliferation study and Mr. T. Kanazawa for performing the numerical simulations discussed in this article.

\section{REFERENCES}

1. B. D. Ratner, et al. Biomedical Engineering Desk Reference, Academic Press, 2009.

2. A. Milella, R. Di Mundo, F. Palumbo, P. Favia, F. Fracassi, R. d'Agostino, Plasma Process. Polym. 6 (2009) 460.

3. P. K. Chu, J. Y. Chen, L. P. Wang, and N. Huang, Mat. Sci. Eng. R 36 (2002) 143.

4. J. Ehlbeck, U. Schnabel, M. Polak, J. Winter, Th. von Woedtke, R. Brandenburg, T. von dem Hagen, and K.-D. Weltmann, J. Phys. D: Appl. Phys. 44 (2011) 013002.

5. M. Laroussi, Plasma Process. Polym. 2 (2005) 391.

6. H. Yasuda, T. Miura, H. Kurita, K. Takashima, and A. Mizuno, Plasma Process. Polym. 7 (2010) 301.

7. A. Postgate1, B. Saunders1, J. Tjandra, J. Vargo, Endoscopy 39 (2007) 361.

8. E. Stoffels, Contrib. Plasma Phys. 47, (2007) 40.

9. J Raiser and M Zenker, J. Phys. D: Appl. Phys. 39 (2006) 3520.

10. G. Lloyd, G. Friedman, S. Jafri, G. Shultz, A. Fridman, and K. Harding, Plasma Process. Polym. 7 (2010) 194.

11. D.-S. Lee, Y. Moriguchi, A. Myoui, H. Yoshikawa, and S. Hamaguchi, J. Phys. D: Appl. Phys. 45 (2012) 372001.

12. K. Masuda, Undergraduate Thesis, School of Engineering, Osaka University (2010).

13. A. Alberti, L. Bolognini, D. Macciantelli, and M. Caratelli, Res. Chem. Intermed. 26 (2000) 253.

14. R. Sensenig, S. Kalghatgi, E. Cerchar, G. Fridman, A. Shershevsky, B. Torabi, K. P. Arjunan, E. Podolsky, A. Fridman, G. Friedman, J. A. Clifford and A. D. Brooks: Annals of Biomedical Engineering. 39 (2011) 674.

15. S. Ikawa, K. Kitano, and S. Hamaguchi, Plasma Process. Polym. 7 (2010) 33. 